\title{
THE LOWER CENTRAL SERIES OF THE GROUP
}

$$
\left\langle x, y: x^{p}=1\right\rangle
$$

JOHN P. LABUTE

\begin{abstract}
In this paper we determine the Lie algebra associated to the lower central series of the group $\left\langle x, y: x^{p}=1\right\rangle, p$ a prime.
\end{abstract}

1. Statement of results. Let $F$ be the free group on $x_{1}, x_{2}$ and let $G=F / R$, where $R=\left(x_{1}^{p}\right)$ is the normal subgroup of $F$ generated by $x_{1}^{p}, p$ a prime. If $\mathfrak{R}$, $g$ are, respectively, the graded Lie algebras associated to the lower central series of $F, G$ (cf. [1], [7], [9]), then $\mathfrak{Z}$ is the free Lie algebra (over $\mathbf{Z}$ ) on $\xi_{1}, \xi_{2}$, the images of $x_{1}, x_{2}$, respectively, in $\mathfrak{L}_{1}(=F /[F, F])$. If $U$ is the enveloping algebra of $\mathfrak{L}$, we view $\mathfrak{L}$ as a left $U$-module via the adjoint representation ad: $U \rightarrow \operatorname{End}_{\mathbf{z}}(\mathfrak{L})$ and identify $\mathfrak{L}$ with its image in $U$ under the canonical injection $\mathfrak{L} \rightarrow U$.

THEOREM. If $\mathfrak{r}$ is the kernel of the canonical surjection $\mathfrak{L} \rightarrow \mathfrak{g}$, then $\mathfrak{r}$ is generated, as an ideal of $\mathfrak{L}$, by the elements

$$
p \xi_{1}, \quad\left[\operatorname{ad}\left(\lambda \xi_{1}\right) \xi_{2}, \operatorname{ad}\left(\lambda \xi_{1}^{n(p-1)+p}\right) \xi_{2}\right] \quad(\lambda \in U, n \geqslant 0) .
$$

This theorem answers a special case of a question posed by Magnus concerning power relations (cf. [9, p. 374]). As a by-product of the proof, we determine the dimensions of the homogeneous components $g_{n}$ for $n \geqslant 2$ as vector spaces over the field $F_{p}$ of $p$-elements. This was done in [2] by entirely different methods.

2. Preliminaries. Let $M$ be the Magnus algebra on $X_{1}, \ldots, X_{n}$, i.e., the algebra of noncommutative formal power series in $X_{1}, \ldots, X_{n}$ with coefficients in $\mathbf{Z}$. Let $\left(t_{1}, \ldots, t_{n}\right)$ be an $n$-tuple of positive real numbers $t_{i}>0$, and define a function

$$
w: M \rightarrow \mathbf{R}_{\geqslant 0} \cup\{+\infty\}
$$

by

$$
\begin{aligned}
& w\left(\sum a_{i_{1} \cdots i_{k}} X_{i_{1}} \cdots X_{i_{k}}\right) \\
& \quad=\operatorname{Inf}\left\{t_{i_{1}}+\cdots+t_{i_{k}}+v_{p}\left(a_{i_{1} \cdots i_{k}}\right): a_{i_{1} \cdots i_{k}} \neq 0\right\},
\end{aligned}
$$

where $v_{p}$ is the $p$-adic valuation on $\mathbf{Z}\left(v_{p}(p)=1\right)$. For each real number

Received by the editors June 22, 1976.

AMS (MOS) subject classifications (1970). Primary 20F35, 20F40; Secondary 17B35, 17B65.

Key words and phrases. One relator group, lower central series, Lie algebra. 
$\alpha \geqslant 0$ let $I_{\alpha}=\{u \in M: w(u) \geqslant \alpha\}$. Then $I_{\alpha} \subseteq I_{\beta}$ if $\alpha \geqslant \beta$, and $I_{\alpha} I_{\beta} \subseteq I_{\alpha+\beta}$ which implies that $I_{\alpha}$ is an ideal of $M$. Since $p \in I_{1}$, we have $p I_{\alpha} \subseteq I_{\alpha+1}$. Let $I_{\alpha}^{+}=\cup_{\beta>\alpha} I_{\beta}$, let $g_{\alpha}(M)=I_{\alpha} / I_{\alpha}^{+}$, and let

$$
\operatorname{gr}(M)=\underset{\alpha>0}{\bigoplus} \mathfrak{g r}_{\alpha}(M) \text {. }
$$

Then $\operatorname{gr}(M)$ is a graded algebra over $\mathbf{F}_{p}$ and $\pi$, the image of $p$ in $\operatorname{gr}_{1}(M)$, is transcendental over $\mathbf{F}_{p}$. Moreover, if $\vec{\xi}_{i}$ is the image of $X_{i}$ in $\operatorname{gr}_{1}(M)$, then $\operatorname{gr}(M)$ is the free associative algebra over $\mathbf{F}_{p}[\pi]$ on $\bar{\xi}_{1}, \ldots, \bar{\xi}_{n}$.

If $F$ is the free group on $x_{1}, \ldots, x_{n}$, then the mapping of $F$ into the group of units of $M$ which sends $x_{i}$ to $1+X_{i}$ is injective (cf. [1], [7], [9]), and we use this injection to identify $F$ with a subgroup of the group of units of $M$. For any real number $\alpha>0$, let $F_{\alpha}=\left(1+I_{\alpha}\right) \cap F$. Then the $F_{\alpha}$ are subgroups of $F$ with

(i) $\cup_{\alpha>0} F_{\alpha}=F$,

(ii) $F_{\alpha} \subseteq F_{\beta}$ if $\alpha \geqslant \beta$,

(iii) $\left[F_{\alpha}, F_{\beta}\right] \subseteq F_{\alpha+\beta}$, where $[H, K]$ is the subgroup of $F$ generated by the commutators $[x, y]=x^{-1} y^{-1} x y$ with $x \in H, y \in K$. Let

$$
F_{\alpha}^{+}=\bigcup_{\beta>\alpha} F_{\beta} \text {. }
$$

Then $F_{\alpha}^{+}$is a normal subgroup of $F$ with $F_{\alpha} / F_{\alpha}^{+}$abelian; in fact, $\left[F_{\alpha}, F_{\beta}\right] \subseteq$ $F_{\alpha}^{+}$for any $\beta>0$. Let $\mathrm{gr}_{\alpha}(F)$ be the abelian group $F_{\alpha} / F_{\alpha}^{+}$, written additively, and let $j_{\alpha}: F_{\alpha} \rightarrow \mathrm{gr}_{\alpha}(F)$ be the canonical surjection. If $\xi=j_{\alpha}(x)$, $\eta=j_{\beta}(y)$, then

$$
[\xi, \eta]=j_{\alpha+\beta}([x, y])
$$

uniquely defines an element $[\xi, \eta] \in \mathrm{gr}_{\alpha+\beta}(F)$, and this bracket operation yields a Lie algebra structure (over $\mathbf{Z}$ ) on the graded abelian group $\operatorname{gr}(F)=$ $\bigoplus_{\alpha>0} \mathfrak{g r}_{\alpha}(F)$, (cf. [1], [8]). Since $x^{p} \in F_{\alpha}^{+}$if $x \in F_{\alpha}$, we have that $\operatorname{gr}(F)$ is a Lie algebra over $\mathbf{F}_{p}$. Now the inclusion $F \subseteq M$ induces a mapping

$$
\theta: \operatorname{gr}(F) \rightarrow \operatorname{gr}(M), \quad x\left(\bmod F_{\alpha}^{+}\right) \rightarrow x-1\left(\bmod I_{\alpha}^{+}\right)
$$

which is an injective Lie algebra homomorphism (over $\mathbf{F}_{p}$ ) of $\operatorname{gr}(F)$ into the underlying Lie algebra of $\operatorname{gr}(M)$, i.e.,

$$
\theta([\xi, \eta])=\theta(\xi) \theta(\eta)-\theta(\eta) \theta(\xi)=[\theta(\xi), \theta(\eta)] .
$$

We use this map to identify $\operatorname{gr}(F)$ with its image in $\operatorname{gr}(M)$.

For $\alpha>0$ let $\varphi(\alpha)=\min (p \alpha, \alpha+1)$ and let $P_{\alpha}: \mathfrak{g r}_{\alpha}(M) \rightarrow \mathrm{gr}_{\varphi(\alpha)}(M)$ be the map defined by

$$
P_{\alpha}(\eta)= \begin{cases}\eta^{p} & \text { if } \alpha<1 /(p-1) \\ \eta^{p}+\pi \eta & \text { if } \alpha=1 /(p-1) \\ \pi \eta & \text { if } \alpha>1 /(p-1)\end{cases}
$$

If $\eta=j_{\alpha}(y) \in \operatorname{gr}_{\alpha}(F)$, then $P_{\alpha}(\eta)=y^{p}\left(\bmod F_{\alpha}^{+}\right)$. The Lie algebra $\operatorname{gr}(F)$ is the smallest graded $\mathbf{F}_{p}$-Lie subalgebra of $\operatorname{gr}(M)$ stable under the $P_{\alpha}$ and 
containing $\bar{\xi}_{1}, \ldots, \bar{\xi}_{n}$ (cf. [8]). If $t_{1}=\cdots=t_{n}=1$, then $\left(F_{n}\right)_{n>1}$ is the lower $p$-central series of $F$, i.e. $F_{1}=F, F_{n+1}=F_{n}^{p}\left[F, F_{n}\right]$ (loc. cit.).

3. Proof of theorem. In the notation of $\$ 2$, we take $n=2, t=t_{1}=t_{2}=$ $1 /(p-1)$. We let $C_{n} F$ denote the $n$th term of the lower central series of $F$; we have $C_{1} F=F, C_{n+1} F=\left[F, C_{n} F\right]$. The inclusion $C_{n} F \subseteq F_{n t}$ defines a Lie algebra homomorphism (over $\mathbf{Z}) \psi: \mathfrak{L} \rightarrow \operatorname{gr}(F)$ whose kernel is $p \mathfrak{L}$ and whose image is the free Lie algebra $\overline{\mathfrak{L}}$ on $\bar{\xi}_{1}, \bar{\xi}_{2}$ over $\mathbf{F}_{p}$.

Let $S$ be the normal subgroup of $F$ generated by $s=\left[x_{1}^{p}, x_{2}\right]$. If

$$
\mathfrak{S}=\bigoplus_{n>1} \mathfrak{g}_{n},
$$

where $\mathfrak{S}_{n}$ is the image of $S \cap C_{n} F$ in $\mathfrak{I}_{n}$, we have $\mathfrak{g}_{1}=0$ and $\mathfrak{S}_{n}=\mathfrak{r}_{n}$ for $n \geqslant 2$. We therefore have $\mathrm{r}=\mathrm{r}_{1} \oplus \mathfrak{g}$ with $\mathrm{r}_{1} \cong \mathbf{Z}$ generated by $p \xi_{1}$. Now let

$$
\mathfrak{S}^{\prime}=\bigoplus_{\alpha>0} \mathfrak{g}_{\alpha}^{\prime},
$$

where $\mathfrak{g}_{\alpha}^{\prime}$ is the image of $S \cap F_{\alpha}$ in $\mathfrak{g r}_{\alpha}(F)$. The image of $s=\left[x_{1}^{p}, x_{2}\right]$ in $\mathrm{gr}_{3 t}(F)$ is

$$
\sigma=\pi\left[\bar{\xi}_{1}, \bar{\xi}_{2}\right]+\operatorname{ad}\left(\bar{\xi}_{1}\right)^{p} \bar{\xi}_{2}
$$

in fact, by an identity of P. Hall (cf. [3]),

$$
\left[x_{1}^{p}, x_{2}\right]=\left[x_{1}, x_{2}\right]^{p} c_{3}^{p} \cdots c_{p}^{p} c_{p+1}
$$

with $c_{l} \in C_{l} F$ and $\operatorname{ad}\left(\bar{\xi}_{1}\right)^{p} \bar{\xi}_{2}$ equal to the image of $c_{p+1}$ in $\operatorname{gr}_{(p+1) t}(F)$.

LEMMA 1. Given $k \geqslant 1, m \geqslant 2, v \in C_{m} F$, there exists $u \in S \cap F_{k+m t} \cap$ $C_{m} F$ such that

$$
u \equiv v^{p^{k}}\left(\bmod C_{m+1} F\right)
$$

Proof. If $z_{1}, \ldots, z_{n} \in F$ and $\operatorname{ad}(x) y=[x, y]$, then

$$
\left(\operatorname{ad}\left(z_{1}\right) \cdots \operatorname{ad}\left(z_{n}\right) s\right)^{p^{k-1}} \equiv\left(\operatorname{ad}\left(z_{1}\right) \cdots \operatorname{ad}\left(z_{n}\right) \operatorname{ad}\left(x_{1}\right) x_{2}\right)^{p^{k}}\left(\bmod C_{n+3} F\right)
$$

and $\left(\operatorname{ad}\left(z_{1}\right) \cdots \operatorname{ad}\left(z_{n}\right) s\right)^{p^{k-1}} \in S \cap F_{k+(n+2) t} \cap C_{n+2} F$, from which the lemma immediately follows.

LEMMA 2. $\psi(\mathfrak{g})=\mathfrak{g}^{\prime} \cap \overline{\mathfrak{I}}$.

Proof. Since $S \cap C_{n} F \subseteq S \cap F_{n t}$ we have $\psi(\mathfrak{g}) \subseteq \mathfrak{g}^{\prime} \cap \overline{\mathfrak{\Omega}}$. Now let $\zeta \in$ $\left(\mathfrak{I}^{\prime} \cap \overline{\mathfrak{L}}\right)_{\alpha}, \zeta \neq 0$, and let $z \in S \cap F_{\alpha}$ with image in $\mathrm{gr}_{\alpha}(F)$ equal to $\zeta$. Assume $z$ chosen so that the integer $m$ with $z \in C_{m} F, z \notin C_{m+1} F$ is as large as possible. If $\alpha=n t$, we have $m \leqslant n$. If $m<n$, then

$$
z \equiv \prod_{i=1}^{q} v_{i}^{p^{k_{i}}}\left(\bmod C_{m+1} F\right)
$$

with $v_{i} \in C_{m} F$ and $k_{i}+m t>\alpha$. By Lemma 1 , there is an element $u \in S \cap$ $F_{\alpha}^{+} \cap C_{m} F$ such that 


$$
u \equiv \prod_{i=1}^{q} v_{i}^{p^{k_{i}}}\left(\bmod C_{m+1} F\right)
$$

Then $z^{\prime}=u^{-1} z \in C_{m+1} F$ and $z^{\prime} \equiv z\left(\bmod F_{\alpha}^{+}\right)$. Hence we must have had $m=n$. If $\rho$ is the image of $z$ in $\mathcal{L}_{n}=C_{n} F / C_{n+1} F$, then $\rho \in \mathfrak{S}_{n}$ and $\psi(\rho)=\zeta$.

Let $\mathfrak{L}^{\prime}$ be the $\mathbf{F}_{p}[\pi]$-Lie subalgebra of $\operatorname{gr}(M)$ generated by $\bar{\xi}_{1}, \bar{\xi}_{2}$. Then (cf. [4, Theorem $\left.\left.4^{\prime}\right]\right) \mathfrak{I}^{\prime}$ is the ideal of $\mathfrak{L}^{\prime}$ generated by $\sigma$. Let $\bar{U}$ be the enveloping algebra of $\overline{\mathfrak{L}}$, i.e. the $\mathbf{F}_{p}$-subalgebra of $\operatorname{gr}(M)$ generated by $\bar{\xi}_{1}, \bar{\xi}_{2}$. Since $p g r_{n}(F) \subseteq \mathfrak{I}_{n}$ for $n \geqslant 2$, the theorem is an immediate consequence of the following lemma.

LEMMA 3. $\overline{\mathfrak{L}} \cap \mathfrak{g}^{\prime}$ is the ideal of $\overline{\mathfrak{L}}$ generated by the elements

$$
\left[\operatorname{ad}\left(\lambda \bar{\xi}_{1}\right) \bar{\xi}_{2}, \operatorname{ad}\left(\lambda \bar{\xi}_{1}^{n(p-1)+p}\right) \bar{\xi}_{2}\right] \quad(\lambda \in \bar{U}, n \geqslant 0) .
$$

Proof. If $\lambda \in \operatorname{gr}(M)$, there are $\lambda_{0}, \lambda_{1}, \ldots, \lambda_{n} \in \bar{U}$ such that $\lambda=\lambda_{0}+\pi \lambda_{1}$ $+\cdots+\pi^{n} \lambda_{n}$. Then, if ad: $\operatorname{gr}(M) \rightarrow \operatorname{End}_{F_{p}[\pi]}\left(\mathfrak{L}^{\prime}\right)$ is the adjoint representation of $\mathfrak{R}^{\prime}$, we have

$$
\begin{aligned}
\operatorname{ad}(\lambda) \sigma= & \operatorname{ad}\left(\lambda_{0}\right) \operatorname{ad}\left(\bar{\xi}_{1}\right)^{p} \bar{\xi}_{2} \\
& +\sum_{i=1}^{n} \pi^{i} \operatorname{ad}\left(\lambda_{i-1}+\lambda_{i} \bar{\xi}_{1}^{p-1}\right)\left[\bar{\xi}_{1}, \bar{\xi}_{2}\right]+\pi^{n+1} \operatorname{ad}\left(\lambda_{n}\right)\left[\bar{\xi}_{1}, \bar{\xi}_{2}\right]
\end{aligned}
$$

Hence $\operatorname{ad}(\lambda) \sigma \in \overline{\mathfrak{L}}$ iff $\lambda_{i-1}+\lambda_{i} \bar{\xi}_{i}^{p-1}(1 \leqslant i \leqslant n)$ and $\lambda_{n}$ annihilate $\left[\bar{\xi}_{1}, \bar{\xi}_{2}\right]$, i.e.

$$
\operatorname{ad}\left(\lambda_{i-1}+\lambda_{i} \bar{\xi}_{1}^{p-1}\right)\left[\bar{\xi}_{1}, \bar{\xi}_{2}\right]=\operatorname{ad}\left(\lambda_{n}\right)\left[\bar{\xi}_{1}, \bar{\xi}_{2}\right]=0 .
$$

But this holds iff for $0 \leqslant i \leqslant n$,

$$
\lambda_{n-i}=\sum_{j=0}^{i}(-1)^{j} \rho_{i-j} \bar{\xi}^{j(p-1)}
$$

with $\operatorname{ad}\left(\rho_{j}\right)\left[\bar{\xi}_{1}, \bar{\xi}_{2}\right]=0$. It follows that the elements $\operatorname{ad}\left(\rho \bar{\xi}_{1}^{n(p-1)}\right) \operatorname{ad}\left(\bar{\xi}_{1}^{P}\right) \bar{\xi}_{2}$ with $n \geqslant 0, \rho \in \bar{U}, \operatorname{ad}(\rho)\left[\bar{\xi}_{1}, \bar{\xi}_{2}\right]=0$ generate $\overline{\mathfrak{L}} \cap \mathfrak{S}^{\prime}$ as a vector space over $\mathbf{F}_{p}$ since $\mathfrak{g}^{\prime}=\{\operatorname{ad}(\lambda) \sigma: \lambda \in \operatorname{gr}(M)\}$. Now a result of [6] states that the annihilator of $\left[\bar{\xi}_{1}, \bar{\xi}_{2}\right]$ in $\bar{U}$ is the left ideal of $\bar{U}$ generated by the elements $\left(\operatorname{ad}(\lambda)\left[\bar{\xi}_{1}, \bar{\xi}_{2}\right]\right) \lambda$ $(\lambda \in \bar{U})$. Hence $\overline{\mathfrak{L}} \cap \mathfrak{I}^{\prime}$ is the ideal of $\bar{\Omega}$ generated by the elements

$$
\begin{aligned}
\operatorname{ad}((\operatorname{ad}(\lambda) & {\left.\left.\left[\bar{\xi}_{1}, \bar{\xi}_{2}\right]\right) \lambda \bar{\xi}_{1}^{n(p-\imath)}\right) \operatorname{ad}\left(\bar{\xi}_{1}^{p}\right) \bar{\xi}_{2} } \\
& =\left[\operatorname{ad}\left(\lambda \bar{\xi}_{1}\right) \bar{\xi}_{2}, \operatorname{ad}\left(\lambda \bar{\xi}_{1}^{n(p-1)+p}\right) \bar{\xi}_{2}\right]
\end{aligned}
$$

with $n \geqslant 0, \lambda \in \bar{U}$. Q.E.D.

Since $\mathrm{r}_{n} \supseteq p \operatorname{prr}_{n}(F)$ for $n \geqslant 2$ we see that $\mathrm{g}_{n}$ is a vector space over $\mathbf{F}_{p}$ for $n \geqslant 2$. To obtain a formula for its dimension we use the fact that for $\alpha \geqslant(p+1) t$,

This yields

$$
\mathfrak{S}_{\alpha}^{\prime}+\overline{\mathfrak{R}}_{\alpha}=\mathfrak{R}_{\alpha}^{\prime}
$$




$$
\begin{gathered}
\text { THE GROUP }\left\langle x, y: x^{p}=1\right\rangle \\
\operatorname{dim}\left(\mathfrak{S}_{\alpha}^{\prime} \cap \overline{\mathfrak{I}}_{\alpha}\right)=\operatorname{dim} \mathfrak{S}_{\alpha}^{\prime}+\operatorname{dim} \overline{\mathfrak{I}}_{\alpha}-\operatorname{dim} \mathfrak{R}_{\alpha}^{\prime},
\end{gathered}
$$

and hence that

$$
\operatorname{dim} \mathfrak{g}_{n}=\operatorname{dim}\left(\mathfrak{R}_{n t}^{\prime} / \mathfrak{g}_{n t}^{\prime}\right)
$$

for $n \geqslant p+1$, as we see using the fact that

$$
\psi\left(\mathfrak{I}_{n}\right)=\overline{\mathfrak{R}}_{n t} \cap \mathfrak{S}_{n t}^{\prime}
$$

Using results of [4] we have

$$
\operatorname{dim} \mathfrak{L}^{\prime} / \mathfrak{g}^{\prime}=\operatorname{dim} \mathfrak{L}^{\prime} / \mathfrak{t}^{\prime},
$$

where $\mathfrak{t}^{\prime}$ is the ideal of $\mathfrak{L}^{\prime}$ generated by $\tau=\operatorname{ad}\left(\bar{\xi}_{1}\right)^{p} \bar{\xi}_{2}$. But

$$
\mathfrak{L}^{\prime} / \mathfrak{t}^{\prime} \cong(\overline{\mathfrak{L}} / \mathfrak{f}) \otimes_{\mathbf{F}_{p}} \mathbf{F}_{p}[\pi] \text {. }
$$

where $\mathfrak{f}$ is the ideal of $\overline{\mathfrak{L}}$ generated by $\tau$. Hence, if $h_{n}=\operatorname{dim} \overline{\mathfrak{I}}_{n t} / \mathfrak{I}_{n t}$, we have for $n \geqslant p+1$,

$$
\operatorname{dim} g_{n}=\sum_{k=1}^{n} h_{n}
$$

where (cf. [5])

$$
n h_{n}=\sum_{d \mid n} \mu(n / d)\left(\sum_{0<i<[d /(p+1)]}(-1)^{i} \frac{d}{d-p i}\left(\begin{array}{c}
d-p i \\
i
\end{array}\right) 2^{d-(p+1) i}\right) .
$$

By inspection, the above formula for $\operatorname{dim} g_{n}$ also holds for $2 \leqslant n \leqslant p$.

\section{REFERENCES}

1. N. Bourbaki, Eléments de mathématique. Groupes et algèbres de Lie, Chapter 2, Hermann, Paris, 1972.

2. A. Gaglione, Factor groups of the lower central series for special free products, J. Algebra 37 (1975), 172-185.

3. P. Hall, $A$ contribution to the theory of groups of prime power order, Proc. London Math. Soc. 36 (1934), 29-95.

4. J. Labute, Algèbres de Lie et pro-p-groupes définis par une seule relation, Invent. Math. 4 (1967), 142-158. MR 36 \# 1581.

5. On the descending central series of groups with a single defining relation, J. Algebra 4 (1970), 16-23. MR 40 \#4342.

6. __ Free Lie algebras as modules over their enveloping algebras, Proc. Amer. Math. Soc. (to appear).

7. M. Lazard, Sur les groupes nilpotents et les anneaux de Lie, Ann. Ecole Norm. Sup. 71 (1954), 101-190. MR 19, 529.

8. , Groupes analytiques p-adiques, Inst. Hautes Études Sci. Publ. Math. No. 26 (1965), 389-603. MR 35 \# 188.

9. A. Magnus, A. Karass and D. Solitar, Combinatorial group theory, Pure and Appl. Math., Vol. 13, Interscience, New York, 1966. MR 34 \# 7617.

Department of Mathematics, McGill University, Montreal, Canada 